\title{
Metodología para la determinación de características del viento y evaluación del potencial de energía eólica en Túquerres - Nariño
}

\author{
Methodology for the determination of wind characteristics and assessment of wind \\ energy potential in Túquerres - Nariño
}

\section{Metodologia para a determinação das características do vento e a avaliação do potencial de energia eólica em Túquerres -Nariño}

\author{
Francisco Eraso-Checa ${ }^{1}$ \\ Edisson Escobar-Rosero ${ }^{2}$ \\ Diego Fernando Paz \\ Carlos Morales ${ }^{4}$
}

Recibido: junio de 2017

Aceptado: noviembre de 2017

Para citar este artículo: Eraso-Checa, F., Escobar-Rosero, E., Paz, D.F., y Morales, C. (2018). Metodología para la determinación de características del viento y evaluación del potencial de energía eólica en Túquerres-Nariño. Revista Científica, 31(1), 19-31. Doi: https://doi.org/10.14483/23448350.12304

\section{Resumen \\ El mundo experimenta un aumento constante en la demanda de energía eléctrica. Una alternativa para suplir dicha demanda consiste en la implementación de energías renovables. En Colombia, desde la apa- rición de la Ley 1715 se ha desarrollado una política de incentivos para la integración de nuevos proyec- tos en este tipo de energías. Por ello, es importante adelantar estudios con datos reales para que even- tualmente puedan ser implementados. En este artícu- lo se presenta el análisis del potencial de generación eólica de la sabana de Túquerres, ubicada en el de- partamento de Nariño, Colombia. El potencial se obtuvo a partir de la medición de la velocidad del viento entre los meses de junio y diciembre del año 2015. Los datos se analizaron estadísticamente se- gún una medida de tendencia central, distribución}

de frecuencias y distribución de Weibull para la normalización de datos dispersos. Finalmente, se calculó la densidad de potencia a partir del modelo de una turbina eólica de eje horizontal y se simuló la curva de generación eléctrica de la zona. La velocidad de viento promedio de la zona es de $4,4 \mathrm{~m} / \mathrm{s}$ y la densidad de potencia encontrada es igual a 3,47 W/m2. Palabras clave: densidad de potencia, distribución del viento, generación de energía, energía eólica, turbina eólica, velocidad del viento.

\begin{abstract}
The world is living a steady increase in the electric power demand, an alternative power generation different to conventional is the renewable energy. With the appearance of the Law 1715, Colombia has an incentives policy for the integration of new projects
\end{abstract}


in renewable energies. Because of that, is important to develop studies with real data in the field of the potential of renewable energy resources which can be implemented. This article presents the analysis of the wind generation potential of Túquerres Savanna, located in the department of Nariño. The potential was obtained from the measurement of the wind speed, during the period between the months of June and December of the year 2015. The data were analyzed statistically according to a measure of central tendency, frequency distribution and Weibull distribution for the normalization of scattered data; finally, the power density was calculated according to a horizontal axis wind turbine and the electrical generation potential of the area was simulated. The average wind speeds are 4,4 $\mathrm{m} / \mathrm{s}$ and the power density founded is $3,47 \mathrm{~W} / \mathrm{m} 2$. Keywords: energy generation, eolic energy, eolic turbine, power density, wind distribution, wind speed.

\section{Resumo}

O mundo está experimentando um aumento constante da procura de electricidade. Uma alternativa para atender a essa demanda é a implementação de energia renovável. Com o advento da Lei 1715, Colômbia desenvolve uma política de incentivos para a integração de novos projetos nesse tipo de energia. Portanto, é importante para fazer avançar os estudos com dados reais para eventualmente, ser implementado. Este artigo apresenta a análise do potencial de geração eólica da sabana de Tuquerres, localizado no departamento de Nariño. O potencial foi obtido a partir da medição da velocidade do vento entre Junho e de Dezembro de 2015. Os dados foram estatisticamente analisados como uma medida da tendência central, distribuição de frequência e distribuição de Weibull para a normalização dos dados dispersos ; finalmente, a densidade de potência é calculado a partir do modelo de uma área de geração de energia eólica de eixo horizontal da curva de potência foi simulado. A área média da velocidade do vento é de $4,4 \mathrm{~m} / \mathrm{s}$ e a densidade de energia é encontrada igual a 3,47 W/m2.

Palavras-chaves: densidade de potência, distribuição do vento, energia eólica, geração de energia, turbina de vento, velocidade do vento.

\section{Introducción}

Colombia experimenta un incremento en la demanda de energía eléctrica debido principalmente a la expansión demográfica y al crecimiento industrial. Una alternativa para cubrir la demanda de esta, teniendo en cuenta la sostenibilidad y confiabilidad del Sistema Nacional Interconectado (SIN), es integrar las energías renovables y aprovechar que Colombia cuenta con diversos recursos energéticos renovables que pueden ayudar a la generación eléctrica requerida para soportar el crecimiento tanto del sector residencial como industrial.

El viento es una de esas fuentes renovables. Su fuerza se aprovecha para la generación de energía eólica mediante los sistemas aerogeneradores. La respuesta eléctrica de un determinado tipo de aerogenerador depende de las condiciones de viento de una zona específica; a partir de la dirección se define la ubicación del aerogenerador y con la velocidad se determina el potencial eólico del viento expresado en potencia por unidad de área (López, 2012). Este parámetro es necesario para alimentar el modelo del aerogenerador y predecir la cantidad de energía eléctrica que se puede generar en dicha zona.

En este documento se analiza el potencial eólico de la sabana de Túquerres, un municipio ubicado en el departamento de Nariño, Colombia, cuya geografía y condiciones climáticas aparentan ser favorables para el aprovechamiento del viento.

\section{Fundamento Teórico}

\section{Condiciones ambientales de la sabana de Túquerres}

La cabecera municipal de Túquerres se ubica sobre la parte alta de la sabana perteneciente al altiplano de Túquerres e Ipiales, atravesado por los ríos Sapuyes y Guáitara. Esta es la altiplanicie más alta de Colombia al pie del volcán Azufral con un promedio de alturas superior a los 2900 msnm y 
se localiza a $72 \mathrm{~km}$ de San Juan de Pasto, capital del departamento de Nariño (Alcaldía de Túquerres, 2015).

El clima está modificado por diferentes factores ya que, pesar de encontrarse en la zona tórrida o intertropical, posee un promedio de temperaturas que oscila entre los 9 y los $13^{\circ} \mathrm{C}$, situación que es determinada por la altura. El promedio anual de lluvias es de $900 \mathrm{~mm}$, valor que aumenta a medida que se desciende hacia el piedemonte de la cordillera occidental en el Pacífico (Alcaldía de Túquerres, 2015). El comportamiento de la curva del viento es mono modal con los mayores valores entre los meses de julio, agosto y septiembre, siendo el mes de agosto el que mayor promedio presenta con $5, .4 \mathrm{~m} / \mathrm{s}$; mientras que los valores medios mínimos se encuentran en el mes de abril con 3,3 m/s (Alcaldía de Túquerres, 2011).

\section{Energía eólica}

El viento es el aire en movimiento causado por la diferencia de temperatura que se encuentra en diferentes puntos de la tropósfera. La energía solar calienta el aire de manera desigual haciendo que las masas de aire caliente asciendan y el aire frio descienda en un movimiento circundante que puede ser suave o fuerte. Esta situación depende de la velocidad, la cual a su vez depende de la altura en la que se encuentre el movimiento del aire (Eraso-Checa, Erazo y Escobar, 2017).

Para la zona ecuatorial a la cual pertenece el municipio de Túquerres, se sitúa una franja de claro predominio de "vientos de componente Este, Nordeste en el hemisferio Norte y Sudeste en el hemisferio Sur. Se trata de los vientos alisios, vientos bastantes constantes y regulares que recorren estas latitudes a una velocidad media de $20 \mathrm{~km} / \mathrm{h}^{\prime \prime}$ (Gonzáles, 2012).

En esencia, la energía eólica aprovecha la energía cinética producida por el viento para hacer girar un sistema de aspas que permiten obtener energía eléctrica mediante el uso de un generador. La electricidad puede consumirse en tiempo real, acumularse para su posterior consumo o transportarse para ser suministrada en otros territorios (Escudero-López, 2011).

\section{Sistemas eólicos}

Un aerogenerador o un conjunto de aerogeneradores conectados a una carga de manera directa o a través de un conversor DC/AC establecen un sistema eólico; estos pueden funcionar de manera independiente o estar conectados a la red comercial. Sus aplicaciones más comunes se orientan al área de telecomunicaciones y a sistemas aislados para viviendas. El generador transforma la energía del viento en corriente directa de 12 o 24 voltios DC que puede entregarse a la carga, a un conversor DC/AC o almacenarse en un banco de baterías. El sistema cuenta con un regulador electrónico de voltaje que vigila el estado de carga de las baterías, distribuye la corriente y compensa las pérdidas de tensión en la línea de conducción (Chinchila-Sánchez, 2001).

La energía eólica es competitiva con respecto a fuentes convencionales de energía como la hídrica y la térmica (Escudero-López, 2011). En la actualidad, se construyen grandes parques eólicos con generadores de 1 a 2 megavatios de potencia (70 m de diámetro y torres de más de 150 metros de altura). España, Alemania y Dinamarca son los países que presentan un mayor crecimiento con instalaciones anuales que superan los 2500 megavatios (Pintor González, 2014).

\section{Aerogenerador de eje horizontal}

Las turbinas eólicas de eje horizontal tienen el eje del rotor principal y el generador eléctrico en la parte superior de una torre y deben apuntar en dirección al viento (Moragues y Rapallini, 2003). Tienen varios componentes como: el rotor que convierte la fuerza del viento en energía rotatoria del eje, una caja de engranajes aumenta la velocidad y un generador transforma la energía del eje en energía eléctrica (Impsa, 2016). 


\section{Potencia suministrada por un aerogenerador de eje horizontal}

La potencia suministrada por un aerogenerador de eje horizontal depende de la superficie barrida por la hélice, es decir, de la longitud de las palas y es independiente del número de las mismas.

La energía que origina el viento es energía cinética causada por la masa del aire en movimiento, como se observa en la ecuación 1.

$$
\mathrm{E}_{\mathrm{c}}=\frac{1}{2} \mathrm{mv}^{2}
$$

Donde $\mathrm{m}$ es la masa del aire en $\mathrm{Kg}$ y $\mathrm{v}$ es la velocidad instantánea del viento $(\mathrm{m} / \mathrm{s})$ como se observa en la ecuación 2.

$$
m=\Phi \cdot V
$$

Donde $\Phi$ es la densidad del aire igual a 1,25 $\mathrm{kg} / \mathrm{m}^{3}$, y $\vee$ es el volumen del cilindro barrido, representado en la ecuación 3.

$$
\mathrm{V}=\mathrm{AL}
$$

Donde A es la superficie barrida, L la longitud del cilindro y t el tiempo, como se observa en la ecuación 4.

$$
L=v t
$$

De esta forma, la energía cinética se representa mediante la ecuación 5 .

$E_{c}=\frac{1}{2} \Phi \cdot$ V $\cdot v^{2}=\frac{1}{2} \Phi \cdot$ A. L. $v^{2}=\frac{1}{2} \Phi \cdot$ A.v.t. $v^{2}=\frac{1}{2} \Phi \cdot$ A. $v^{3} \cdot t$

La ecuación 6. determina la potencia teórica del aerogenerador.

$$
P=\frac{E_{c}}{t}=\frac{1}{2} \Phi \cdot \mathrm{A} \cdot v^{3}
$$

Si el área barrida transversalmente por el viento es circular, como ocurre en todas las máquinas eólicas de eje horizontal, la ecuación 7. permite calcular dicha área.

$$
A=\Phi \cdot \frac{D^{2}}{4}
$$

Siendo D el diámetro; por lo que la potencia disponible se observa en la ecuación 8.

$$
P=\frac{\pi}{8} \cdot \Phi \cdot D^{2} \cdot v^{3}
$$

\section{Caracterización del aerogenerador}

La energía almacenada en el viento es muy elevada pero esta no se aprovecha en su totalidad. Albert Betz demostró en 1919 que la extracción de la energía del viento hace que este reduzca su velocidad. Según el teorema de Betz, la energía máxima que podrá captarse del viento para que el rendimiento sea el máximo es el 59\% de la energía cinética del viento; esto representa la primera perdida del rendimiento final de la turbina (Villanueva y Marcello, 2016). La potencia que se puede extraer del viento con un aerogenerador está determinada por la ecuación 9. (Escudero-López, 2011).

$$
P=C p \cdot \frac{\Phi}{2} \cdot \frac{\pi \cdot D^{2}}{4} \cdot V^{3}
$$

En donde:

$C p$ : coeficiente de potencia.

$\Phi$ : densidad del aire $\left(\mathrm{Kg} / \mathrm{m}^{3}\right)$.

$V$ : velocidad del viento $(\mathrm{m} / \mathrm{s})$.

D: diámetro de las hélices $(\mathrm{m})$.

Un parámetro importante para caracterizar el funcionamiento de un aerogenerador es la densidad del aire, la cual para Colombia oscilan entre $0,7 \mathrm{Kg} / \mathrm{m}^{3}$ sobre zonas de alta montaña y $1,2 \mathrm{Kg} / \mathrm{m}^{3}$ en zonas ubicadas a nivel del mar. Teniendo en cuenta la información previa, el valor de la densidad del aire a trabajar es $0,7 \mathrm{Kg} /$ $\mathrm{m}^{3}$ el cual corresponde a zonas de alta montaña (Upme, s.f.), semejante al lugar de desarrollo de la investigación. 
Una vez se ha conseguido la densidad del aire para el lugar de ubicación del sistema y se ha conocido el valor de la velocidad promedio del viento, se obtiene la potencia del viento por medio de la ecuación 10.

$$
\frac{P}{A}=\frac{1}{2} \cdot \Phi V^{3}
$$

En donde:

$\frac{P}{A}$ : densidad de potencia $\left(\mathrm{W} / \mathrm{m}^{2}\right)$.

$\Phi$ : densidad del aire $\left(\mathrm{Kg} / \mathrm{m}^{3}\right)$.

$V$ : velocidad del viento $(\mathrm{m} / \mathrm{s})$.

\section{Metodología}

\section{Instalación de la estación meteorológica}

Para la instalación se consideraron los parámetros de la Organización Meteorológica Mundial (OMM), entre los que se encuentran: la ubicación del anemómetro, la cual debe estar a 10 metros de altura con relación al suelo; la resistencia de los postes a vientos fuertes; la ubicación correcta de la veleta mediante el uso de una brújula; el tiempo de muestreo igual a 10 minutos; y la calibración de los instrumentos de medición. Los datos se analizaron teniendo en cuenta el aerogenerador horizontal.

\section{Organización de los datos adquiridos por la estación}

Los datos extraídos de la estación correspondieron a la velocidad y dirección del viento, estos, se organizaron por hora y fecha en una hoja de cálculo, registrando promedios por hora, día, semana y mes.

\section{Medidas de tendencia central de la velocidad del viento}

Se realizó el cálculo de la velocidad del viento media, máxima, mínima, la moda, la mediana y la desviación estándar de los datos recolectados durante el periodo de monitoreo mediante la herramienta de análisis descriptivo de Excel®.

\section{Distribución de frecuencia del viento}

Se realizó la distribución de frecuencias con los datos adquiridos en el periodo de monitoreo, los cuales se organizaron de menor a mayor. Al aplicar la regla de Sturges se obtuvo el número y la amplitud de clases en los que se distribuye la velocidad del viento; se calculó la frecuencia absoluta la cual corresponde al número de ocurrencias en horas de cada intervalo; y se obtuvo la probabilidad de ocurrencia de la velocidad del viento para cada intervalo (esto se observa en la tabla 4).

\section{Densidad de potencia eólica}

La estimación de la densidad del potencial eólico de la sabana de Túquerres para el periodo de monitoreo, se calculó de acuerdo a la ecuación 10. Para este cálculo se tuvo en cuenta el valor de la densidad del viento ( $\Phi$ ) igual a $0,7 \mathrm{Kg} / \mathrm{m}^{3}$ (Upme).

\section{Ajuste de la densidad de potencia con distribu- ción de Weibull}

Se utilizó la función de probabilidad de Weibull (Walpole, 2007) con el fin de realizar un análisis estadístico de los datos de densidad de potencia, debido a que esta ha demostrado tener un buen ajuste con los datos de velocidad del viento. Para calcular la probabilidad de Weibull se determinaron los parámetros de forma y escala ( $\beta$ y $\alpha$ ), obtenidos por medio del análisis de regresión lineal entre los valores de la variable y su probabilidad acumulativa, utilizando la transformada logarítmica y el método de mínimos cuadrados.

\section{Coeficiente de potencia}

A partir de la ecuación 9. se calculó el coeficiente de potencia que determina el valor máximo de 
aprovechamiento de la energía cinética disponible en la sabana de Túquerres. Este valor debe es inferior al límite de Betz.

\section{Análisis matemático del viento en la sabana de Túquerres}

\section{Registro de datos}

El 5 de junio de 2015 se llevó acabo la instalación de la estación meteorológica Meteoagro MA 3081 en la sabana de Túquerres, a una altura de 3533 msnm, en las coordenadas $1^{\circ} 5^{\prime} 29.01^{\prime \prime}$ latitud Norte y $77^{\circ} 40^{\prime} 22.00^{\prime \prime}$ longitud Oeste, correspondiente a la finca San Pedro. El periodo de monitoreo fue de junio a diciembre de 2015. La descarga de información se realizó cada dos semanas, periodo en el cual se supervisó el funcionamiento de la estación.

Para la instalación de la estación se tuvieron en cuenta las normas que establece la OMM y se siguieron sus recomendaciones para la documentación de la información recopilada, organizándola según la descripción de los instrumentos utilizados, la metodología de recolección de información, el periodo de tiempo de recolección de datos, los parámetros monitoreados, las coordenadas y geografía de la zona y los responsables de las mediciones.

\section{Velocidad del viento}

La tabla 1 presenta los datos de velocidad de viento $(\mathrm{m} / \mathrm{s})$ promediados para cada uno de los meses correspondientes al periodo de muestreo. El promedio general por hora es de 4,4 m/s.

Tabla 1. Velocidad promedio del viento por mes $(\mathrm{m} / \mathrm{s})$ (junio-diciembre).

\begin{tabular}{rrrrrc}
\hline Junio & Julio & Septiembre & Octubre & Noviembre & Diciembre \\
\hline 3,74 & 3,65 & 4,26 & 4,85 & 4,64 & 5,26 \\
\hline
\end{tabular}

Fuente: elaboración propia.

\section{Análisis de la velocidad del viento}

Partiendo de la información promedio horaria de la velocidad del viento almacenada por la estación meteorológica cada 10 minutos, se obtuvo la gráfica del comportamiento del viento durante cada mes del periodo de monitoreo y la gráfica total del mismo (figura 1).

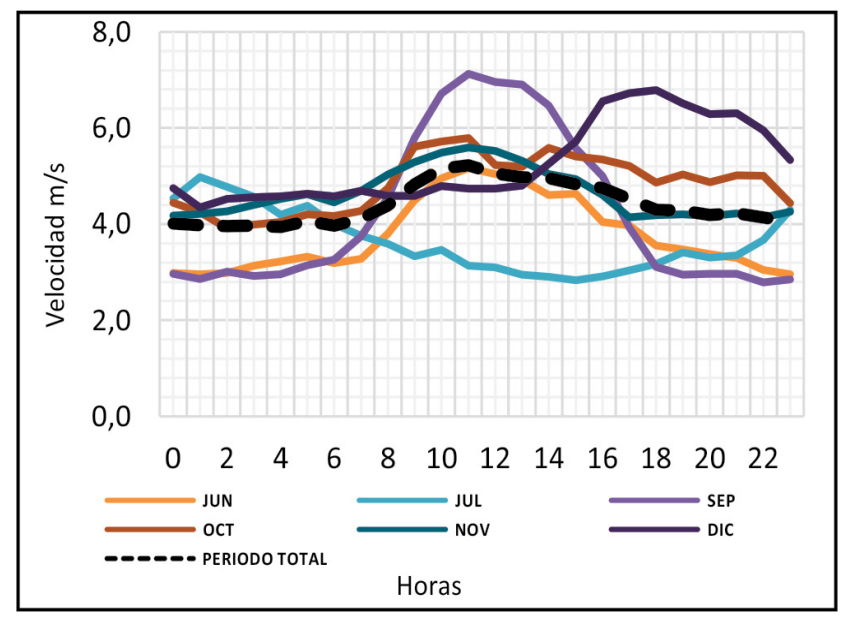

Figura 1. Velocidad horaria del viento durante junio a diciembre del 2015.

Fuente: elaboración propia.

Se observa que en el mes de septiembre se obtuvieron los mejores registros de velocidad, con una velocidad máxima de $7,2 \mathrm{~m} / \mathrm{s}$ promedio hora; el mes con menor velocidad registrada fue julio, donde se presentaron velocidades entre 2,8 y $5 \mathrm{~m} / \mathrm{s}$; para los demás meses la velocidad estuvo por encima del valor requerido para la generación de energía eléctrica, el cual es de $3,5 \mathrm{~m} / \mathrm{s}$. La línea punteada describe el comportamiento de la velocidad promedio hora del viento durante todo el periodo de monitoreo, este permanece constante entre las 12 y las 6 horas con una velocidad de $4 \mathrm{~m} / \mathrm{s}$; a partir de las 7 hasta las 11 horas se incrementa la velocidad hasta alcanzar un valor pico de $5,2 \mathrm{~m} / \mathrm{s}$; por último, se puede observar que entre las $7 \mathrm{y}$ las 18 horas se alcanza una velocidad superior a las $4 \mathrm{~m} / \mathrm{s}$. 


\section{Medidas de tendencia central de la velocidad del viento}

Haciendo uso de la herramienta de análisis descriptivo de Excel® ${ }^{\circledR}$, se realizó el cálculo de la media, máxima, mínima, moda, mediana y desviación estándar de la velocidad del viento monitoreada. En el análisis se presenta la velocidad media mensual del viento y se demuestra que esta se encuentra por encima del valor necesario para la generación de energía eólica. La velocidad máxima se encuentra entre $12,9 \mathrm{~m} / \mathrm{s}$ y 13,9 $\mathrm{m} / \mathrm{s}$ en intervalos cortos de tiempo pero que significan un buen potencial de generación eléctrica (véase tabla 2). La velocidad mínima registrada fue de $1 \mathrm{~m} / \mathrm{s}$.

El cálculo de la moda representa el dato de velocidad con mayor frecuencia, siendo para el mes de junio, julio y septiembre igual $2,7 \mathrm{~m} / \mathrm{s}$, para los meses de octubre y noviembre de $3,7 \mathrm{~m} / \mathrm{s}$ y para diciembre de $3,8 \mathrm{~m} / \mathrm{s}$. La mediana es el valor central en un conjunto de todos los datos ordenados con los datos registrados en la tabla. Por último, se calculó la desviación estándar, la cual indica la variabilidad que tienen los datos con respecto a la media. Se puede notar que la variabilidad durante el periodo de monitoreo no supera el 2,8\%.

Teniendo en cuenta que la altura mínima de un aerogenerador de eje horizontal es de $30 \mathrm{~m}$ sobre el nivel del suelo, se procede a calcular la velocidad media del viento a esta altura a ese nivel aplicando la ecuación 11.

$$
\operatorname{Vm}(z)=\operatorname{Vm}(r) \frac{z^{\propto}}{r}
$$

Donde:

$\operatorname{Vm}(z)$ : velocidad media a la altura $(z)$ de exploración $(\mathrm{m} / \mathrm{s})$.

$\operatorname{Vm}(r)$ : velocidad media a la altura de referencia $(\mathrm{m} / \mathrm{s})$.

$Z$ : altura a la cual se va a explorar $(\mathrm{m})$.

$r$ : altura de referencia $(\mathrm{m})$.

$\propto$ : coeficiente de rigurosidad del sistema

En la tabla 3 se presentan los valores de velocidad del viento en la sabana de Túquerres a 30 metros de altura $(z)$, partiendo de la ubicación de la estación ( $r$ ) y un valor del coeficiente de rugosidad $(\propto)$ igual a 0,1 correspondiente a las características de la zona donde se llevó a cabo el monitoreo de la velocidad del viento.

Tabla 3. Velocidad del viento a 10 y 30 metros de altura.

\begin{tabular}{lllllll}
\hline & & \multicolumn{4}{l}{$\begin{array}{l}\text { Velocidad del } \\
\text { viento }(\mathbf{m} / \mathbf{s})\end{array}$} \\
Mes & $\mathbf{Z}$ & $\mathbf{r}$ & $\propto$ & $\begin{array}{l}\text { Altura } \\
\text { Altura }\end{array}$ & Incremento (\%) \\
& & & & $\mathbf{1 0} \mathbf{~ m t s}$ & $\mathbf{3 0} \mathbf{~ m t s}$ & \\
\hline Jun & & & & 3,8 & 4,2 & $12 \%$ \\
Jul & & & & 3,7 & 4,1 & $12 \%$ \\
Sep & & & & 4,3 & 4,8 & $12 \%$ \\
Oct & 30 & 10 & 0,1 & 4,8 & 5,4 & $12 \%$ \\
Nov & & & & 4,6 & 5,2 & $12 \%$ \\
Dic & & & & 5,3 & 5,9 & $12 \%$ \\
\hline
\end{tabular}

Fuente: elaboración propia.

Tabla 2. Medidas de tendencia central para la velocidad del viento.

\begin{tabular}{crrrrrr}
\hline Mes & Media & Max & $\begin{array}{c}\text { Min } \\
\mathbf{m} / \mathbf{s}\end{array}$ & Moda & Mediana & Desviación estándar (\%) \\
\hline Jun & 3,8 & 12,9 & 1,0 & 2,7 & 3,1 & 2,2 \\
Jul & 3,7 & 12,9 & 1,0 & 2,7 & 3,1 & 2,1 \\
Sep & 4,3 & 13,9 & 1,0 & 2,7 & 3,7 & 2,3 \\
Oct & 4,8 & 12,9 & 1,0 & 3,7 & 4,4 & 2,8 \\
Nov & 4,6 & 13,6 & 1,0 & 3,7 & 4,4 & 1,4 \\
Dic & 5,3 & 13,7 & 1,0 & 3,8 & 4,8 & 1,8 \\
Prom & 4,4 & 13,9 & 1,0 & & & \\
\hline
\end{tabular}

Fuente: elaboración propia. 
En la tabla 3 se observa que a 30 metros de altura la velocidad del viento se incrementa en un $12 \%$, haciendo lo que hace factible la implementación de tecnologías para generación de energía eólica pues las velocidades estarían por encima de los $4 \mathrm{~m} / \mathrm{s}$ y superarían la velocidad necesaria para la generación eólica (Eoliccat, 2016).

\section{Distribución de frecuencias de la velocidad del viento}

Para realizar la distribución de frecuencias de la velocidad del viento se aplicó la regla de Sturges (González, 2005), para lo cual es necesario conocer el rango de datos, el número y el ancho del intervalo; esta distribución se observa en la tabla 4. En esta se encuentran los límites de velocidad del viento inferior ( $\mathrm{Li})$, superior (Ls), la frecuencia relativa ( $\mathrm{fr}$ ) y su sumatoria acumulada ( $\mathrm{Fr}$ ), la frecuencia absoluta (fa) y su sumatoria acumulada $(\mathrm{Fa})$.

Se puede observar que a partir del número de clase tres la producción de energía eólica es de
19.906 horas, las cuales se obtiene mediante la sumatoria de las frecuencias absolutas (fa). Esto significa que el $77 \%$ del tiempo muestreado el sistema eólico genera electricidad.

\section{Histograma de velocidad del viento en la sabana de Túquerres}

La distribución de frecuencias de la velocidad del viento se visualiza en la figura 2. Esta se organiza teniendo en cuenta el número de clases encontradas mediante la regla de Sturges. Se puede observar que la mayor cantidad de datos se encuentra ubicada en la clase cuya tendencia central es $3,7 \mathrm{~m} / \mathrm{s}$.

También se observa que los datos presentan asimetría hacia la derecha, lugar en el que se encuentran las velocidades que superan los $3 \mathrm{~m} / \mathrm{s} .15$ 374 datos se encuentra entre 3 y $6 \mathrm{~m} / \mathrm{s}$ lo que corresponde al 60,1\% del total de las 25581 horas monitoreadas. Lo anterior significa que la zona de estudio presenta un buen potencial para la implementación de energía eólica.

Tabla 4. Distribución de frecuencias mediante la regla de Sturges.

\begin{tabular}{llllllll}
\hline \multirow{2}{*}{ No. } & \multicolumn{2}{l}{ Velocidad } \\
& $\mathbf{L i}(\mathbf{m} / \mathbf{s})$ & $\mathbf{L s}(\mathbf{m} / \mathbf{s})$ & Marca $(\mathbf{m} / \mathbf{s})$ & fa & fr & Fa & Fr \\
\hline 1 & 1,0 & 1,9 & 1,4 & 2107 & 0,082 & 2107 & 0,0824 \\
2 & 1,9 & 2,8 & 2,3 & 3568 & 0,139 & 5675 & 0,2218 \\
3 & 2,8 & 3,7 & 3,2 & 4006 & 0,157 & 9681 & 0,3784 \\
4 & 3,7 & 4,6 & 4,1 & 5535 & 0,216 & 15216 & 0,5948 \\
5 & 4,6 & 5,4 & 5,0 & 3708 & 0,145 & 18924 & 0,7398 \\
6 & 5,4 & 6,3 & 5,9 & 2439 & 0,095 & 21363 & 0,8351 \\
7 & 6,3 & 7,2 & 6,8 & 2003 & 0,078 & 23366 & 0,9134 \\
8 & 7,2 & 8,1 & 7,7 & 942 & 0,037 & 24308 & 0,9502 \\
9 & 8,1 & 9,0 & 8,6 & 747 & 0,029 & 25055 & 0,9794 \\
10 & 9,0 & 9,9 & 9,4 & 236 & 0,009 & 25291 & 0,9887 \\
11 & 9,9 & 10,8 & 10,3 & 74 & 0,003 & 25365 & 0,9916 \\
12 & 10,8 & 11,7 & 11,2 & 36 & 0,001 & 25401 & 0,9930 \\
13 & 11,7 & 12,6 & 12,1 & 98 & 0,004 & 25499 & 0,9968 \\
14 & 12,6 & 13,4 & 13,0 & 49 & 0,002 & 25548 & 0,9987 \\
15 & 13,4 & 14,3 & 13,9 & 23 & 0,001 & 25571 & 0,9996 \\
16 & 14,3 & 15,2 & 14,8 & 10 & 0,000 & 25581 & 1,0000 \\
\hline Total & & & $\mathbf{2 5 5 8 1}$ & $\mathbf{1}$ & $\mathbf{2 5 5 8 1}$ & \\
\hline
\end{tabular}

Fuente: elaboración propia. 


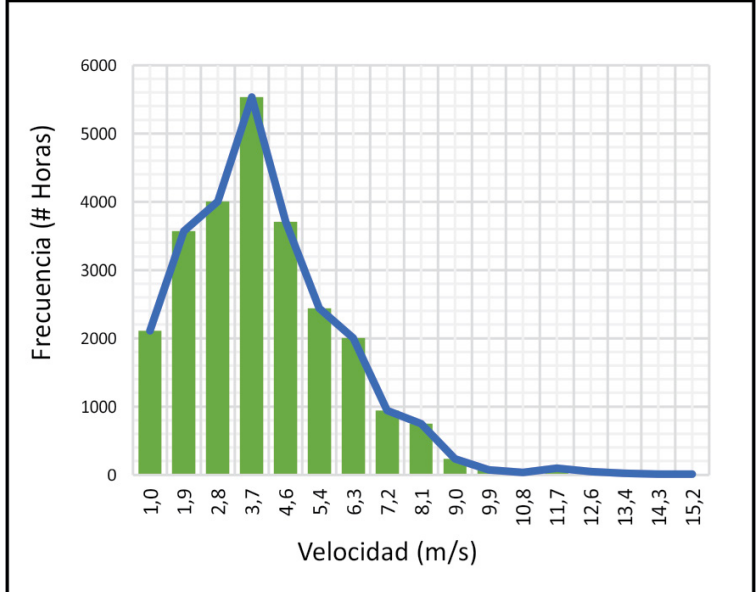

Figura 2. Histograma de la velocidad del viento. Fuente: elaboración propia.

\section{Densidad de potencia eólica}

La estimación de la densidad del potencial eólico de la sabana de Túquerres para el periodo de monitoreo se calcula de acuerdo a la ecuación 10 y se representa en la tabla 5 para cada una de las clases.

Las variaciones que presenta el viento dependen de las condiciones climatológicas de la zona, por esto es necesario el uso de métodos estadísticos para evaluar el aprovechamiento del potencial eólico en la zona. Dada la variabilidad y asimetría del histograma representado en la figura 2, se puede normalizar la función de la probabilidad de distribución de la velocidad del viento a través del análisis de Weibull. Los parámetros para dicho análisis se obtuvieron mediante el análisis de regresión lineal entre los valores de la variable y su probabilidad acumulativa, utilizando la transformada logarítmica y el método de mínimos cuadrados. La función de distribución de acumulación se presenta en la ecuación 12.

$$
W(v)=1-e^{-\frac{v^{\alpha}}{\beta}}
$$

En donde:

$W(v)$ : función de distribución de probabilidad Weibull para la velocidad del viento. $v$ : velocidad del viento.

$\propto$ : parámetro de forma.

$\beta$ : parámetro de escala.

Tabla 5. Densidad de potencia en la sabana de Túquerres.

\begin{tabular}{|c|c|c|}
\hline Vel $(m / s)$ & $\operatorname{Vel}^{3}\left(\mathrm{~m}^{3} / \mathrm{s}^{3}\right)$ & P/A $\quad\left(W / m^{2}\right)$ \\
\hline 1,0 & 1,00 & 0,35 \\
\hline 1,9 & 6,74 & 2,36 \\
\hline 2,8 & 21,44 & 7,50 \\
\hline 3,7 & 49,32 & 17,26 \\
\hline 4,6 & 94,59 & 33,11 \\
\hline 5,4 & 161,47 & 56,51 \\
\hline 6,3 & 254,17 & 88,96 \\
\hline 7,2 & 376,91 & 131,92 \\
\hline 8,1 & 533,92 & 186,87 \\
\hline 9,0 & 729,40 & 255,29 \\
\hline 9,9 & 967,57 & 338,65 \\
\hline 10,8 & 1252,65 & 438,43 \\
\hline 11,7 & 1588,86 & 556,10 \\
\hline 12,6 & 1980,41 & 693,14 \\
\hline 13,4 & 2431,51 & 851,03 \\
\hline 14,3 & 2946,39 & 1031,24 \\
\hline 15,2 & 3529,27 & 1235,24 \\
\hline
\end{tabular}

Fuente: elaboración propia.

Con una transformación de doble logaritmo la ecuación 13 puede ser escrita como:

$\operatorname{Ln}\left(\operatorname{Ln}\left(\frac{1}{1-W(v)}\right)\right)=\propto \operatorname{Ln}(v)-\propto \operatorname{Ln}(\beta$

De esta manera se representa la ecuación 13. en una ecuación lineal descrita en la ecuación 14.

$$
y=\propto x-b
$$

Para la ecuación 14, la pendiente de la recta representa el parámetro de forma $(\propto)$. En esta $Y$ se representa por la ecuación 15 y X por la ecuación 16.

$$
y=\operatorname{Ln}\left(\operatorname{Ln}\left(\frac{1}{1-W(v)}\right)\right)
$$




$$
x=\operatorname{Ln}(v)
$$

A partir de la deducción de la ecuación lineal de la función de probabilidad acumulada de Weibull ((5), se encontraron los parámetros $\alpha$ y $\beta$, en donde $\alpha$ es igual a la pendiente de la recta y $\beta$ es igual al exponencial del cociente entre punto de corte de la recta en el eje Y. Es decir $\alpha=2,2625 ; \beta$ $=5,09$.

La velocidad normalizada del viento se representa en la figura 3. Luego de conocer la probabilidad de Weibull para cada velocidad del viento se estimó la densidad de potencial eólico especifica ajustada de la sabana de Túquerres para los datos obtenidos durante el periodo de monitoreo de acuerdo a la (3, como se muestra en la columna P/A (W[v]) de la tabla 6.

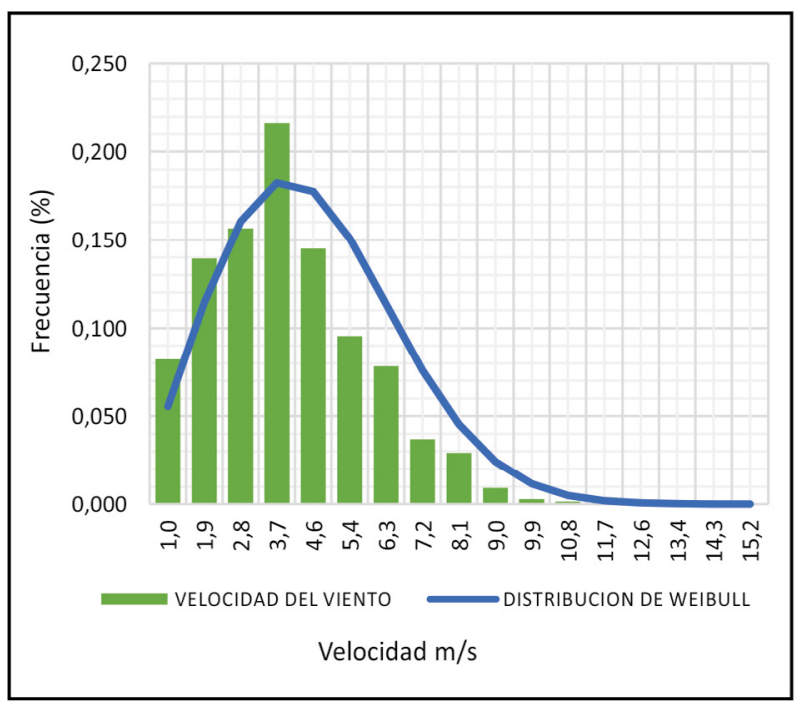

Figura 3. Distribución de Weibull de la velocidad del viento.

Fuente: elaboración propia.

\section{Coeficiente de potencia}

Como la potencia contenida en el viento no puede estar por encima del límite de Betz equivalente al 59,26\% de la energía cinética del viento, la columna P/A (Cp) de la tabla 6 muestra la potencia real disponible para la sabana de Túquerres.
La figura 4 presenta, en amarillo, la densidad de potencia real que puede entregar un aerogenerador de eje horizontal ubicado en la sabana de Túquerres, teniendo en cuenta la componente de velocidad típica de la zona, la cual se relaciona en el eje horizontal. También se observa que dicha curva se encuentra por debajo de la curva de potencia normalizada utilizando la distribución de Weibull, representada por el área azul.

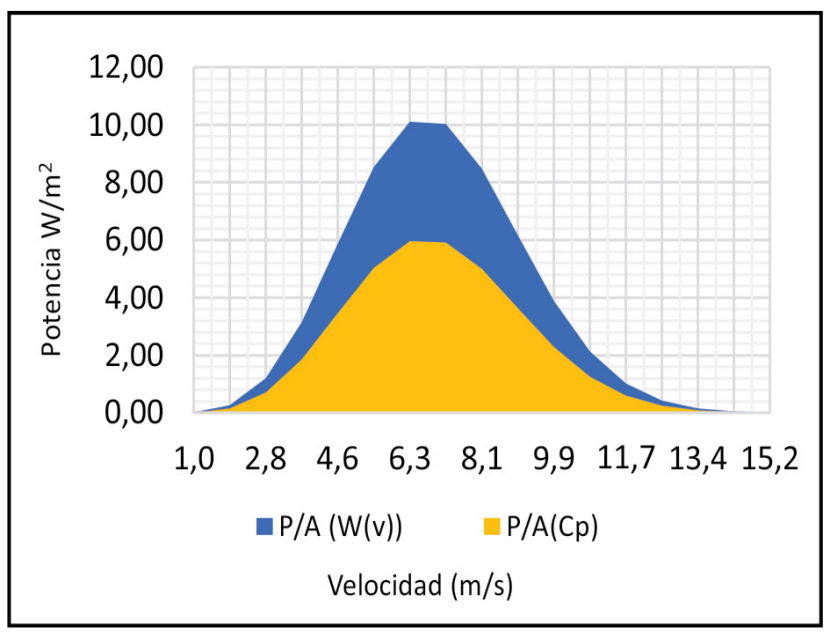

Figura 4. Densidad del potencial eólico en la sabana de Túquerres.

Fuente: elaboración propia.

Estos parámetros de viento se entregaron a un modelo de un aerogenerador de eje horizontal que se presenta en la figura 5. El aerogenerador consta de una entrada de velocidad del viento, una entrada de velocidad del generador, una señal de entrada para el ángulo de paso beta y la salida correspondiente a la potencia mecánica.

La simulación obtenida a la salida del aerogenerador presenta la curva de potencia de la figura 6. La velocidad de arranque inicia a los $3 \mathrm{~m} / \mathrm{s}$, lo que genera una potencia de $16 \mathrm{~W}$; a $12 \mathrm{~m} / \mathrm{s}$ alcanza la potencia nominal de $3,5 \mathrm{~kW}$, Ilegando alcanzar una potencia de $2,7 \mathrm{~kW}$ a una velocidad de $15,2 \mathrm{~m} / \mathrm{s}$. A esta velocidad el aerogenerador cuenta con un sistema de frenado ya que la velocidad nominal es de $12 \mathrm{~m} / \mathrm{s}$. 


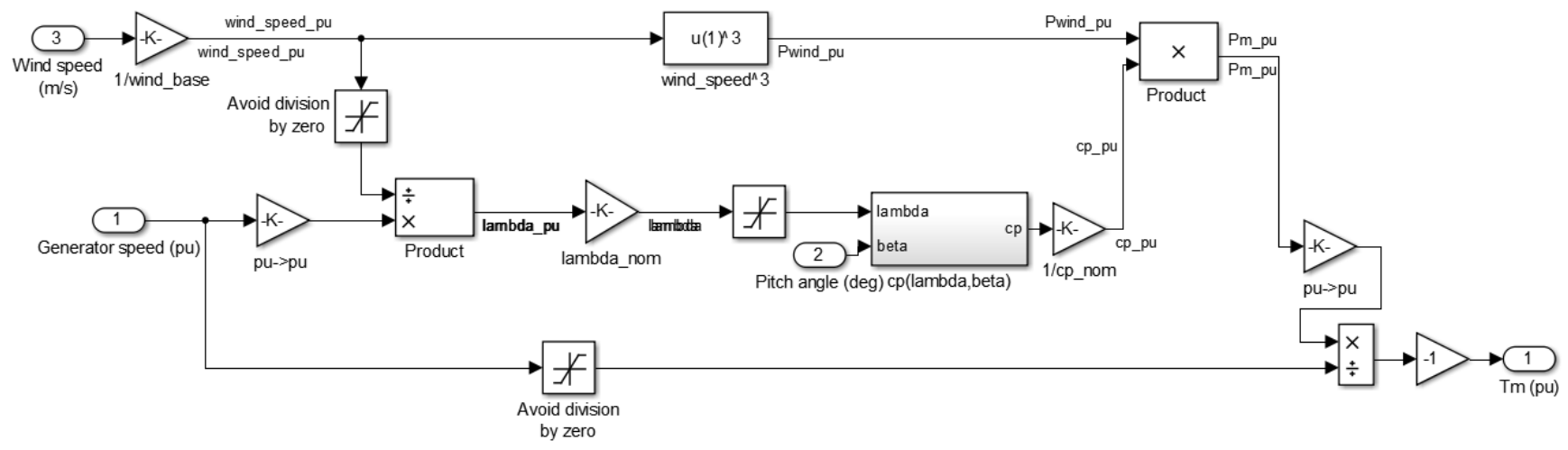

Figura 5. Modelo de la turbina eólica.

Fuente: elaboración propia.

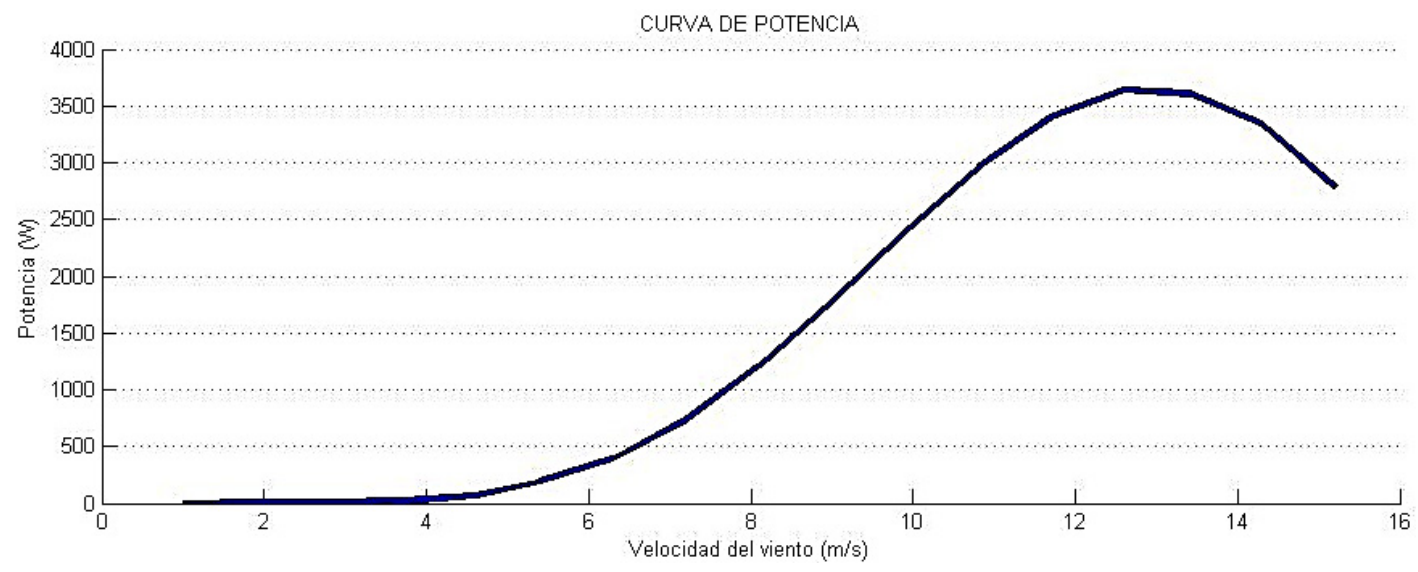

Figura 6. Curva de potencia de la simulación.

Fuente: elaboración propia.

Tabla 6. Densidad del potencial.

\begin{tabular}{|c|c|c|c|c|c|}
\hline Vel $(\mathrm{m} / \mathrm{s})$ & $\left(\mathbf{m}^{3} / \mathbf{s}^{3}\right)$ & $\mathrm{P} / \mathrm{A}\left(\mathrm{W} / \mathrm{m}^{2}\right)$ & P. Weibull & P/A (W(v)) & P/A (Cp) $\left(W / m^{2}\right)$ \\
\hline 1,0 & 1,00 & 0,4 & 0,05555 & 0,02 & 0,01 \\
\hline 1,9 & 6,74 & 2,4 & 0,11436 & 0,27 & 0,16 \\
\hline 2,8 & 21,44 & 7,5 & 0,16051 & 1,20 & 0,71 \\
\hline 3,7 & 49,32 & 17,3 & 0,18250 & 3,15 & 1,86 \\
\hline 4,6 & 94,59 & 33,1 & 0,17747 & 5,88 & 3,47 \\
\hline 5,4 & 161,47 & 56,5 & 0,15099 & 8,53 & 5,03 \\
\hline 6,3 & 254,17 & 89,0 & 0,11362 & 10,11 & 5,96 \\
\hline 7,2 & 376,91 & 131,9 & 0,07602 & 10,03 & 5,92 \\
\hline 8,1 & 533,92 & 186,9 & 0,04535 & 8,48 & 5,00 \\
\hline 9,0 & 729,40 & 255,3 & 0,02415 & 6,17 & 3,64 \\
\hline 9,9 & 967,57 & 338,7 & 0,01148 & 3,89 & 2,29 \\
\hline 10,8 & 1252,65 & 438,4 & 0,00487 & 2,13 & 1,26 \\
\hline 11,7 & 1588,86 & 556,1 & 0,00184 & 1,02 & 0,60 \\
\hline 12,6 & 1980,41 & 693,1 & 0,00062 & 0,43 & 0,25 \\
\hline 13,4 & 2431,51 & 851,0 & 0,00019 & 0,16 & 0,09 \\
\hline 14,3 & 2946,39 & 1031,2 & 0,00005 & 0,05 & 0,03 \\
\hline 15,2 & 3529,27 & 1235,2 & 0,00001 & 0,01 & 0,01 \\
\hline
\end{tabular}

Fuente: elaboración propia. 


\section{Conclusiones}

Una implementación de un sistema eólico de eje horizontal eficiente requiere que la zona de ubicación cuente con una velocidad promedio del viento superior a $3,5 \mathrm{~m} / \mathrm{s}$, debido a que partir de esta velocidad se obtiene un potencial eléctrico significativo. La sabana de Túquerres mostró una velocidad promedio igual a 4,4 m/s durante el periodo evaluado; esto significa que el potencial de generación eólico para esta zona es aprovechable desde el punto de vista técnico. El análisis estadístico realizado indica que el promedio de generación de densidad de potencia es igual a $3,47 \mathrm{~W} / \mathrm{m} 2$.

El trabajo estadístico desarrollado, sumado a la utilización del límite de Betz, permite generar un escenario real de aprovechamiento del potencial eólico de la sabana de Túquerres en el que se contemplan las pérdidas energéticas normales correspondientes al proceso de conversión de la energía. Es de resaltar que sin el uso de estas herramientas se sobrestimaría el potencial de una zona de estudio y los resultados reales no coincidirán con los diseños.

La toma de datos se realizó entre los meses de junio y diciembre del año 2015. Sin embargo, los resultados pueden ser generalizados para todo el año, pues al tratarse de una zona ecuatorial las condiciones ambientales no cambian de manera.

\section{Referencias}

Alcaldía de Túquerres. (2011). Plan básico de ordenamiento territorial municipio de Túquerres. Recuperado de: http:// www.tuquerres-narino.gov.co/apc-aa-files/65663135376634333334353635353636/ POT.pdf

Alcaldía de Túquerres. (27 de febrero de 2015). Unidos por Túquerres. Recuperado de: http:// www.tuquerres-narino.gov.co/documentos municipio.shtml

Chinchila-Sánchez, M. (2001). Control de un sistema de generación eólica de velocidad variable con generador síncrono multipolar de imanes permanentes acoplado a red. Leganés: Universidad Carlos III de Madrid.

Eoliccat. (1 de junio de 2016). ¿Qué fuerza debe tener el viento para accionar los aerogeneradores? Recuperado de: http://eoliccat.net/la-tecnologia/ preguntas-frecuentes/?lang=es

Eraso-Checa, F., Erazo, O. y Escobar, E. (2017). Energía fotovoltaica: modelos y respuestas a condiciones meteorológicas. Pasto, Colombia: Institución Universitaria Cesmag.

Escudero-López, J. (2011). Manual de energía eólica. México D.F.: Mundi-Prensa.

Gonzáles, M. (1 de marzo de 2012). Clima ecuatorial. Recuperado de: http://titulaciongeografia-sevilla.es/contenidos/profesores/ materiales/archivos/2012-04-16CLIMAeCUATORIAL.pdf

González, J.J. (2005). El histograma con la TI92: optimización de clases. Revista de Didáctica de las Matemáticas, 61, 67-72.

Impsa. (2016). Aerogeneradores. Recuperado de: http://www.impsa.com/es/productos/impsawind/SitePages/aerogeneradores. aspx

López, M.V. (2012). Ingeniería de la energía eólica. Barcelona: Marcombo.

Moragues, J. y Rapallini, A. (2003). Energía eólica. Buenos Aires: Instituto Argentino de la Energía General Mosconi.

Pintor González, S. (2014). Energías renovables y desarrollo sostenible $=$ Renewable energy and sustainable development.

Upme. (s.f.). upme.gov.co. Recuperado de: http://www.upme.gov.co/Atlas Viento.htm

Villanueva, Y. y Marcello, R. (2016). Análisis estructural y modal de la hélice de un aerogenerador de $3 \mathrm{~kW}$ de potencia 
mediante simulación numérica computacional. Lima: Pontificia Universidad Católica del Perú.
Walpole, R.E. (2007). Probabilidad y estadística para ingeniería y ciencias. México D.F.: Pearson Educación. 